\title{
Is disruptive mood dysregulation disorder a precursor for borderline personality disorder?
}

\section{Hugo André de Lima Martins}

Unidade do Cérebro, Surubim, Pernambuco, Brazil.

\section{$\bigotimes$}

Hugo André de Lima Martins Rua Antônio Medeiros Sobrinho, 275, Cabaceira, Surubim-PE, Brasil hugomt2001@yahoo.com.br

Edited by:

Marcelo Moraes Valença

Keywords:

Borderline personality disorder

Mood disorders

Disruptive behavior

Child

Adolescent

Anger

Impulsive behavior

\begin{abstract}
Disruptive mood dysregulation disorder in children and adolescents is characterized by chronic irritability, in which outbursts of anger are manifested either verbally or through aggressive behavior. Before the last update of the Diagnostic and Statistical Manual of Mental Disorder (DSM-5), many severely irritable children were diagnosed with bipolar disorder. A borderline personality disorder is characterized by emotional instability, episodes of anger, impulsivity, and irritability; therefore, it is often misidentified as bipolar disorder. The behavior of disruptive mood dysregulation disorder resembles many characteristics of borderline, which diagnosis usually occurs in young adulthood. Is disruptive mood dysregulation disorder a precursor for borderline disorder?
\end{abstract}




\section{Introduction}

E irst described in the DSM-5, disruptive mood dysregulation disorder (DMDD) is frequent among children in pediatric mental health services. ${ }^{1}$ Its main characteristics are severe irritability ${ }^{1,2}$ and recurrent temper outbursts, manifested verbally or behaviorally. The intensity and duration of these events are disproportionate to provocation or some situations, and temper outbursts are inconsistent with the patient developmental level while they occur three or more times per week. The patient also demonstrates notable persistent irritability (e.g., with parents, teachers, and colleagues) or angriness during most of the day.

The first diagnosis should not occur before six or after eighteen. ${ }^{1,2}$ The above-mentioned symptoms must persist for at least 12 months, and patients cannot experience more than three months without symptoms. The symptoms must appear in at least two contexts (i.e., at home, school, or with colleagues), while the patient should not meet the criteria for mania, and symptoms could not occur exclusively during a depressive episode or be explained by severe mental disorder (e.g., autism). ${ }^{1 \cdot 3}$

Previous researchers interpreted recurrent severe irritability as characteristic of bipolar disorder in childhood. 2,4 Due to this, the number of children diagnosed with bipolar disorder increased at the end of the 20th century, and children were medicated with antipsychotics, thus experiencing side effects of these drugs. ${ }^{2,3}$ Therefore, the inclusion of DMDD in the DSM-5 proposed a different classification for children with irritability and anger outbursts.

Diagnosis of bipolar disorder and borderline personality disorder in adults is frequently misunderstood, and other authors had assumed that borderline disorder was included in the bipolar spectrum. ${ }^{5,6}$ Borderline disorder is often diagnosed in late adolescence or young adulthood, and symptoms result from emotional dysregulation, such as irritability, frequent mood swings, impulsivity, anger outbursts, and difficulties in interpersonal relationships. ${ }^{7,8}$ Therefore, DMDD and borderline symptoms overlap, as demonstrated in Figure 1.

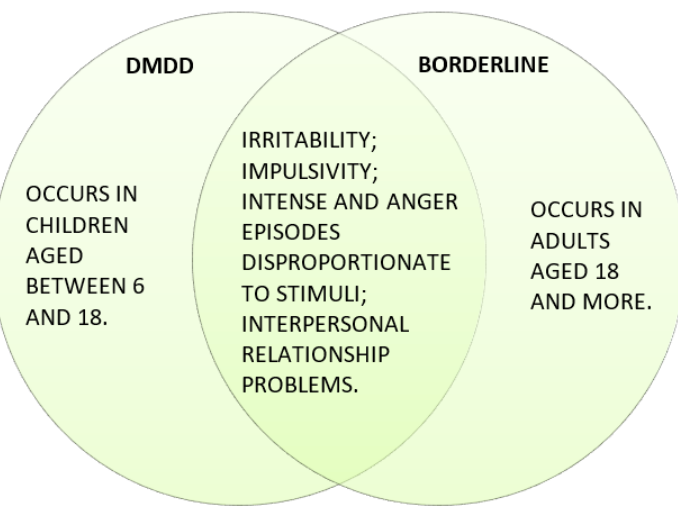

Figure 1. Symptoms overlap between disruptive mood dysregulation disorder (DMDD) and borderline personality disorder.

Considering DMDD diagnosis was described eight years ago in the DSM-5 and symptoms are developed during childhood, we do not have sufficiently follow-up studies to understand the personality of these patients in adulthood. ${ }^{3}$ In addition, a longitudinal study evaluating children with severe mood dysregulation demonstrated the development of anxiety and depressive disorders in adulthood. ${ }^{9}$

Long-term follow-up studies with DMDD children and adolescents are needed to corroborate the possibility that many patients will be diagnosed with borderline disorder in adulthood. Although some researchers hypothesize that DMDD is more prevalent in males and borderline in females, at least in a clinical context ${ }^{8}$, answers to a follow-up would only be available after at least one decade because of behavioral alterations during the transition from childhood to adulthood.'

This hypothesis is important due to the possibility of earlier interventions, especially with dialectical behavioral therapy. This therapy is the gold standard for treating severe emotional dysregulation, such as borderline personality disorder. ${ }^{10}$

Hugo Andre de Lima Martins

https://orcid.org/0000-0002-5216-2074

\section{References}

1. Baweja R, Mayes SD, Hameed U and Waxmonsky JG. Disruptive mood dysregulation disorder: current insights. Neuropsychiatr Dis Treat 2016;12:2115-2124 Doi:10.2147/ndt.S100312

2. American Psychiatric Association. Diagnostic and statistical manual of mental disorders.5th ed. 2013;55(3):220

3. Copeland WE, Shanahan L, Egger H, Angold A and Costello EJ. Adult Diagnostic and Functional Outcomes of DSM-5 Disruptive Mood Dysregulation Disorder. American Journal of Psychiatry 2014;171(6):668-674 Doi:10.1176/appi.ap.2014.13091213

4. Leibenluft E, Cohen P, Gorrindo T, Brook JS and Pine DS. Chronic versus episodic irritability in youth: a community-based, longitudinal study of clinical and diagnostic associations. J Child Adolesc Psychopharmacol 2006;16(4):456-466 Doi:10.1089/ cap.2006.16.456

5. Magill CA. The boundary between borderline personality disorder andbipolardisorder:currentconceptsandchallenges. CanJPsychiatry 2004;49(8):551-556 Doi: 10.1177/070674370404900806

6. Towbin K, Axelson D, Leibenluft $E$ and Birmaher B. Differentiating bipolar disorder-not otherwise specified and severe mood dysregulation. J Am Acad Child Adolesc Psychiatry 2013;52(5):466481 Doi:10.1016/j.jaac.2013.02.006

7. Martins HADL. Is borderline personality disorder the cause of chronic headache? Avanços em Medicina 2021;Doi:10.52329/ avanmed. 15

8. Martins HA, Martins BBM, Mazullo BB, Martins VRR and dos Santos ALB. Borderline and Antisocial Personality Disorders in the Bible. Journal Psychol Psyclother Res. 2021;8:11-21

9. Brotman MA, Schmajuk M, Rich BA, Dickstein DP, Guyer AE, Costello EJ, ... Leibenluft E. Prevalence, clinical correlates, and longitudinal course of severe mood dysregulation in children. Biol Psychiatry 2006;60(9):991-997 Doi:10.1016/i.biopsych.2006.08.042

10. Linehan MM and Wilks CR. The Course and Evolution of Dialectical Behavior Therapy. American Journal of Psychotherapy 2015;69(2):97110 Doi:10.1176/appi.psychotherapy.2015.69.2.97 